\title{
Analyse structurelle des Epithetes de Maurice de La Porte (1571)
}

\author{
Nathalie Gasiglia, ${ }^{1, *}$ \\ ${ }^{1}$ Université de Lille, UMR 8163 - STL - Savoirs Textes Langage, F-59000 Lille, France
}

\begin{abstract}
Résumé. L'élaboration d'une édition numérique des Epithetes de Maurice de La Porte (1571) nous conduit à analyser méticuleusement les patrons de construction de ses articles. Si la majorité contient un substantif en adresse suivi d'une liste d'épithètes applicables à cet item et optionnellement un commentaire terminal, certains articles peuvent s'avérer plus riches. Cette contribution se fixe pour objectif d'analyser les structures d'articles atypiques afin, d'une part, de comprendre ce qui a motivé La Porte à les employer et, d'autre part, de proposer un balisage XML TEI le plus adéquat possible de ce dictionnaire de combinaisons de mots.
\end{abstract}

\begin{abstract}
Structural analysis of Les Epithetes by Maurice de La Porte (1571). In order to develop a digital edition of Les Epithetes by Maurice de La Porte (1571), we meticulously analysed the structural patterns of its entries. Although most comprise a headword followed by a list of epithets applicable to it, sometimes followed by a comment, some entries have further enrichments. The aim of this contribution is to analyse the structure of non-typical entries in order to understand what prompted de La Porte to use them, and to propose the most appropriate XML TEI tag set for this dictionary of collocations.
\end{abstract}

\section{Introduction}

Dans le cadre de l'édition numérique en cours d'un dictionnaire de combinaisons de mots, Les Epithetes de Maurice de La Porte $(1571)^{2}$, nous avons besoin d'inventorier méticuleusement les patrons de construction des 3748 articles compilés ${ }^{3}$. Pour réaliser ce premier dictionnaire d'épithètes en langue française, son auteur a procédé à un relevé méthodique de cooccurrences de substantifs (noms communs et noms propres) et d'épithètes (simples ou polylexicales) au sein des textes de la Pléiade, en particulier ceux de Ronsard. ${ }^{4}$ Son " épithétaire ", pour reprendre le néologisme introduit par le lexicographe ${ }^{5}$, se présente sous la forme d'un ensemble d'articles contenant un substantif en adresse suivi d'une liste de qualifications qui en sont des cooccurrents typiques et parfois d'un commentaire. Mais ce patron de construction, qui semble au premier abord assez simple et régulier, s'avère plus riche, soumis à des variations structurelles quand il y a plusieurs substantifs ou des déterminatifs en adresse ou qu'il y a d'autres unités linguistiques traitées dans l'article, quand la liste des épithètes peut être subdivisée en sous-parties commentées, quand les articles comportent des citations ou des renvois à des textes d'auteurs ou des renvois à d'autres articles du volume, etc. Cette contribution se fixe pour objectif d'analyser les structures d'articles atypiques afin de tenter de comprendre ce qui a motivé le lexicographe et de déterminer comment il est opportun de traiter ces articles dans le cadre d'une édition électronique enrichie d'occurrences attestées dans la Pléiade (cf. Pouey-Mounou \& Gasiglia (2015 et à paraître) et Gasiglia \& PoueyMounou (2017)), qui présuppose un balisage XML TEI ${ }^{6}$ des articles le plus adéquat possible.

\footnotetext{
*nathalie.gasiglia@univ-lille3.fr
} 
Dans cette édition, outre la restitution du texte des Epithetes, notre ambition est de donner accès aux attestations des cooccurrences relevées dans les textes de la Pléiade qui, si elles sont antérieures à 1571, peuvent avoir été des sources pour le lexicographe. Cette édition enrichie permettra donc à ceux qui veulent chercher à distinguer les sources des réemplois et, plus largement, à ceux qui sont curieux de voir les cooccurrents en usage, de disposer de citations et d'accéder aux contextes plus longs à partir de celles-ci, ce qui documentera finement les études littéraires ou lexicales à venir (cf. Gasiglia \& Pouey-Mounou (2017, §§ 2.1. et 3.2.1.)).

\section{Patron type des articles}

Les Epithetes étant un dictionnaire de combinaisons de mots, la structure des articles sert donc ce projet en associant à des substantifs des listes d'épithètes qui les accompagnent communément en langue ou au moins chez les auteurs de la Pléiade, ou des qualifications que La Porte a souhaité rendre disponibles pour du réemploi (donc dans un but pédagogique, pour former les futurs auteurs à bien écrire). Ces qualifications peuvent être des adjectifs monolexicaux ou des séquences plus complexes. Chaque article (cf. fig. 1) débute donc par un composant d'adressage (cf. § 3.1), qui est composé dans un corps un peu plus gros que la suite de l'article et ponctué d'un point, et qui présente le ou les substantif(s) ${ }^{7}$ pour le(s)quel(s) des épithètes sont ensuite proposées en une liste. Au sein de cette dernière, chaque qualification est en italique et est suivie d'une virgule, sauf la dernière, qui l'est d'un point.

Si le cœur du projet dictionnairique est la présentation de cooccurrences lexicales choisies, La Porte s'est également donné les moyens de pouvoir formuler divers types de commentaires. Un composant en général terminal d'article leur est dédié. Optionnel, il est écrit en romain (s.v. Auerne en fig. 1 et cf. § 4). Dans quelques cas, des renvois sont proposés afin de compléter le traitement local d'un substantif avec les épithètes proposées dans un autre article, donc concernant un autre substantif (s.v. Aube et Auette en fig. 1 et cf. $\S 5.2$ ).

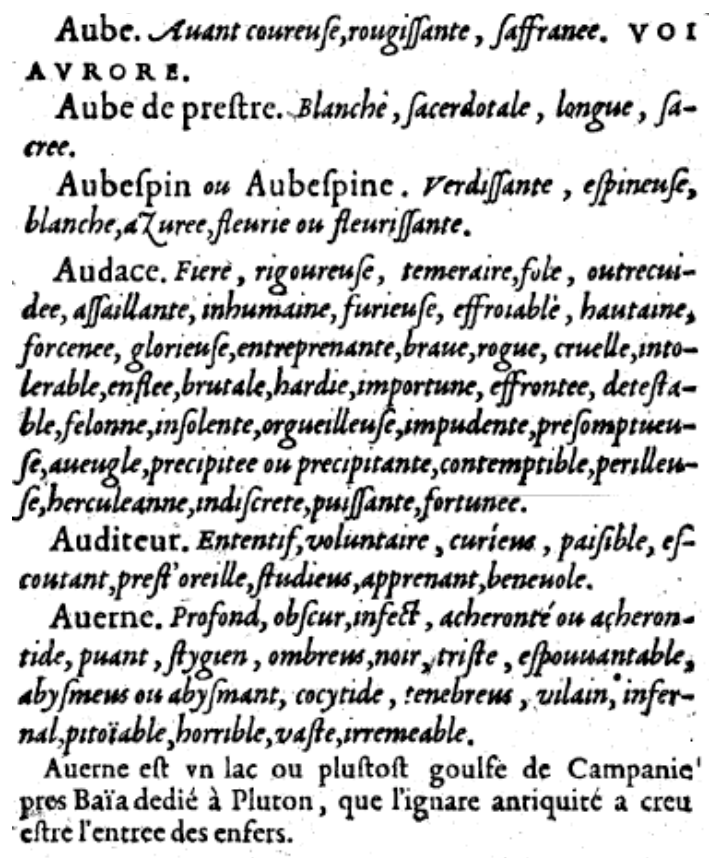

Auette. Blonde. CHERCHE A B ILLE.

Fig. 1. Articles Aube, Aube de prestre, Aubespin ou Aubespine, Audace, Auditeur, Auerne et Auette.

Il peut aussi y avoir d'autres substantifs introduits (diminutifs ou formes au féminin, $\mathrm{cf}$. $\S \S 3.2$ et 3.3 ) et des citations (cf. $\S 4.2$ ). 
Hors des articles, mais à leur proximité, 15 notes marginales imprimées figurent dans le texte des Epithetes. Elles servent le plus souvent à localiser des citations ou des propos rapportés : s.v. Baleine (« Plin.li.9. chap.3.», cf. fig. 2), Corbeau ou Corbin (« [Pline] Liure 7. chap. 48. »), Galien (« Mũstere en sa Cosmographie, liu. 4.fueillet 1227. au bas. »), Mine ou Miniere (« Pline.liu 33.chap.6 »), Perdris (« Pline liu. 10. chap. 33. »), Satyres (« [Pline] Liure 7. chap.2. »), Sauge (« Mathiole en ses cõment. sur Diosc. liure3.chapit.34. »), Souabes ou Souaues (« [lule Cesar] Liure 4. de ses Cõmẽtaires. »), Tisane ou Ptisane (" [Pline] Liure 18. chap.7. »), Tortue (« [Pline] Liure 9. chap.10. ») et Turcs («Belon en ses obseruat. liu.3. chap.16.»). Mais certaines fournissent des précisions sur des éléments des commentaires : s.v. Colysee («Le Stade contient 125. pas, qui sont 625.pieds»), Heliogabale ( « Le Cirque estoit le lieu ou ils faisoient les ioustes \& combats. ») et Laïs (« Vne drachme valloit trois sols six deniers. »). Et 1, enfin, est métalinguistique : elle est placée en vis-à-vis des articles Aduocat, Eaque ou Eaque et Egine. (« Auiourdhui le frãa̧ois ortographe admet raremẽnt les diphtõgues, de sorte que celui qui en use indifferãmẽt ne doit estre accusé d'ignorãce. », cf. § 3.2 .2 et fig. 3).

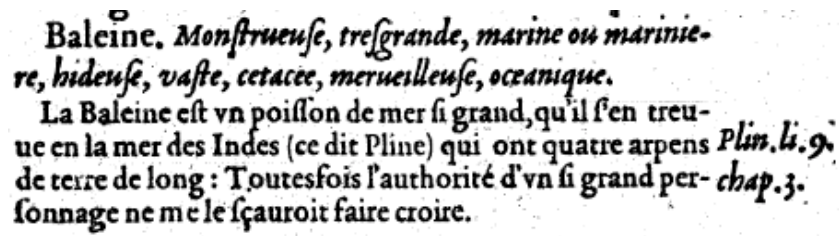

Fig. 2. Note marginale en vis-à-vis de l'article Baleine.

\section{Plusieurs substantifs traités dans un même article}

Ainsi que cela a déjà été indiqué, la structure régulière d'un article consiste à présenter un substantif en adresse puis à formuler à son sujet deux types de propos : d'une part, une liste de qualifications typiques et, d'autre part, optionnellement, un commentaire rédigé. Il arrive assez souvent que le composant d'adressage contienne plusieurs mots, soit une séquence formant un tout, soit deux substantifs qui partagent le même traitement et qui sont alors coadressés, c'est-à-dire présentés l'un après l'autre en début d'article. Il arrive également qu'après le traitement du ou des substantif(s) en adresse principale, un autre substantif soit présenté puis traité : il s'agit alors d'un sous-adressage, le nouveau substantif n'étant pas une adresse principale mais subordonnée, celle d'un sous-article imbriqué dans le premier. Dans ces deux cas, adressage principal et subordonné, les unités linguistiques appartiennent à la macrostructure (ici double) du dictionnaire, elles donnent accès aux articles principaux ou enchâssés qui les traitent. Mais il existe aussi des cas de traitement microstructurel des substantifs, dans lesquels il ne se fait pas sous la forme d'une liste d'épithètes suivie optionnellement d'un commentaire, mais est réduit à la monstration de ce nouveau substantif associée à une information morphologique, l'indication du fait que le substantif introduit est un diminutif ou une forme au féminin de l'item ou d'un des items en adresse principale. Dans tous les cas les adressages sont typographiquement marqués par une taille de caractères plus grande que celle des autres composants des articles.

Dans chacun des cas d'adressages multiples, la question est de savoir si La Porte a simplement voulu mentionner ces substantifs ou s'il souhaitait les faire hériter de tout ou partie du traitement proposé pour la ou les unité(s) linguistique(s) en adresse principale.

\subsection{Plusieurs mots dans le composant d'adressage principal}

Deux cas de figure peuvent motiver que le premier composant d'un article, celui qui l'introduit et qui sert à son classement alphabétique dans la liste des articles, comporte plusieurs mots. Il peut y avoir quelques cas de substantifs polylexicaux, souvent des noms propres, qui ne constituent qu'un substantif en adresse. Il peut y avoir un substantif accompagné d'un 
sélecteur de sens (Aube de prestre en fig. 1), et dans ce cas encore il n'y a qu'un substantif objet du traitement qui suit. Ce sont ces cas de substantifs uniques présentés avec plusieurs mots que nous traiterons d'abord, avant de prendre en compte ceux où il y a plusieurs substantifs en adresse principale d'article, avec un coadressage de ces items afin de bien les placer sur un pied de quasi-égalité (imparfaite, puisqu'il y a nécessairement un ordre de mention).

\subsubsection{Substantifs en adresses principales polylexicaux ou désambiguïsés}

Une partie des substantifs à la nomenclature sont des noms propres polylexicaux (Ance martien, Des Autels), des noms associés à des titres (Charles 9. à present Roi de France) ou combinant prénom et nom (Anne de Mõmoranci, Catherine de Medicis), mais il peut aussi y avoir des titres de fonctions (Bedeau de l'Vniuersité, Fermier du Prince, Recteur de l'vniuersité), des noms d'instances (Barreau des aduocats) ou liés à la religion (Le sainct Esprit, S. Iean I'Euangeliste, Iesus Christ, Marie mere de Dieu). 33 adresses de l'épithétaire sont des noms de cet ordre.

Souvent, ailleurs, la présence d'une adresse principale polylexicale est motivée par le souhait de désambiguïser des homographes : l'article Accord ou Accordance (avec coadressage, cf. § 3.1.2) précède celui consacré à Accord de sons, comme Amorce précède Amorce d'Haquebute, Aspic précède Aspic Serpent, Aube précède Aube de prestre (cf. fig. 1), etc. Mais que la présence d'une adresse polylexicale soit motivée par un souhait de différenciation d'homographes ou non, il arrive que celle-ci soit en usage sous cette forme complexe uniquement (Mouches à miel) ou en concurrence avec l'emploi du premier nom seulement (Instrumens de musique). Par ailleurs, quand il y a coadressage, les deux noms têtes sont séparés par ou et le déterminatif est mis en facteur commun : Materas ou Mattelas a coucher équivaut à Materas a coucher ou Mattelas a coucher.

Pour les 54 substantifs homographes différenciés de ceux qui les précèdent, divers types de sélecteurs de sens sont employés :

- une classe taxinomique directement postposée au substantif ou séparée de lui par une virgule ou concaténée par de et le plus souvent sans majuscule initiale (Aspic Serpent, Bresil bois, Liure de poix, Lote poisson, Maquereau poisson, Masse, herbe, Perche poisson, Raye poisson, Rue herbe),

- une glose (Port signifiant contenãce),

- une destination, ce à quoi sert le référent nommé (Carriere pour Course, Fers qu'on met aux pieds, Instrumens de musique, Materas ou Mattelas a coucher, Materas à tirer, Mortier à bastir, Mouches à miel, Naquet de ieu de paume, Oraison pour priere, Poix à peser, Presse d'Imprimerie, Question pour torture, Tour pour finesse, Tour pour circuit, Tour pour reuolution, Trenchees de guerre, Voile à voguer),

- un détenteur typique (Aube de prestre, Fumees de Cerf, Mine d'aucun, Motte soit d'homme ou de femme),

- une relation entre une partie constituante et un tout (Amorce d'Haquebute, Cep de Vigne, Cordes d'instrumens, Corps Humain, Greues des iambes, Palme ou Paulme de la main, Son de fourment, Tuyau ou Tuiau de bled) ou entre un tout et une partie remarquable (Chapeau de fleurs),

- une localisation, qui peut être véritablement localisatrice (Mine qu'on fait soubs la terre), ou plus conventionnelle, dans des termes d'astronomie (Chien coeleste et Ourse celeste), ou dans le nom d'un animal importé d'Amérique du Nord par les colons espagnols vers 1500 (Coq d'Inde),

- une relation établie entre des objets de même nature (Accord de sons),

- une sélection sémantique de types de compléments de noms (Monstre de quelque chose,

Patron de quelque chose),

- l'indication d'un contenant (Bouillon du pot), 
- et des relations moins aisées à expliciter, dans des noms de végétaux (Bourse ou Boursette au pasteur et Chardon beneist), dans la dénomination d'un mouvement corporel (Bransle de corps) ou dans un nom d'objet (Chef d'œuure).

Dans 19 cas, la mention d'un sélecteur de valeur n'est pas liée à la présence d'un autre substantif homographe en adresse. C'est le cas pour des substantifs associés

- à leur destination (Greffe dequoi on ente, Paelle à frire, Parquet ou lon plaide, Ruben de teste, Toüaille à mains),

- à leur détenteur typique (Panetiere d'vn berger, Penil d'homme ou de femme, Teint d'homme ou de femme),

- à l'expression du tout pour une partie constituante (Coutre d'vne charrue, Gonds d'vne porte, Mords de bride, Nacre de perles) ou d'une partie remarquable pour un tout (Fusee de poudre a canon, Guillee d'eau, Lauage d'eau),

- à leur objet (Tutelle d'enfans),

- ou à autre chose encore (Bord de l'eau, Cours d'eau, Manne du ciel).

\subsubsection{Coadressages de plusieurs substantifs}

Sur les 3748 articles des Epithetes, 625 comportent des coadressages. Parmi ceux-ci, 267 correspondent à des variantes graphiques des mêmes substantifs (comme "AAge ou Age. ", « Agneau ou Aigneau. », « Aneth ou Enneth. » ou « Ani ou Anis. »). Parmi les 358 autres occurrences, il s'observe de rares cas de synonymie («Rame ou Auiron. » ou « Vefuage ou Viduité. »). Mais les coadressages concernent aussi des dérivations concurrentes, dont, souvent, l'une seulement est restée en usage. Il peut s'agir de bases ou de conversions alternatives à des suffixations, ou de suffixations différentes (« Accord ou Accordance. », « Assailleur ou Assaillant. », " Auare ou Auaricieux. », « Fruits ou Fruitages. » ou « Pasteur, Pastre ou Pastoureau. »). Si les référents des substantifs coadressés précédents sont les mêmes, il existe aussi des cas de coadressage de noms d'entités parentes mais distinctes ("Ambassade ou Ambassadeur. ", « Artizan ou Artiste. », « Crin ou Criniere. », « Fain ou Famine. » ou « Fueillard ou Fueille. »), des noms animaux dont la variation en genre correspond à la différence de sexe («Cane ou Canart. »), ou encore des diminutifs, construits avec les suffixes -ette et -ul/-il (« Able ou Ablette. », "Faucille ou Faulx. », les diminutifs ablette et faucille figurant respectivement en seconde et en première position).

Le coadressage des substantifs les mettant sur le même plan, le traitement déployé ensuite vaut donc de manière équivalente pour l'une et l'autre de ces unités linguistiques. Il sera néanmoins intéressant d'observer quelles cooccurrences sont effectivement dans les textes de la Pléiade et ont donc pu être relevées par le lexicographe.

\subsection{Adressages subordonnés}

Lors du traitement d'un substantif en adresse principale, il arrive qu'un autre substantif soit présenté et associé à des informations qui lui sont propres. Il s'agit alors d'une adresse subordonnée qui introduit un article enchâssé au sein du premier. Le plus souvent la forme de ces adressages est régulière, mais dans quelques cas leur patron rédactionnel est libre.

\subsubsection{Adressages subordonnés à patrons rédactionnels libres}

Quelques substantifs en adresses subordonnées ne sont pas introduits dans une formulation régulière, mais dans des énoncés variés. Par exemple, s.v. Copie, après la liste d'épithètes, figure « De Copie nous auons Copiste. Plumeteur, diligent, laborieus, vigilant. », où l'on trouve l'adresse subordonnée Copiste, qui est présentée comme dérivée de copie mais sans explication de la relation morphosémantique, et une liste d'épithètes qui est propre à copiste, et, en même position, s.v. Biche, « Son faon ou fan est appellé Bicheteau. Delitieus, viande roiale, tendre, exquis. », qui 
explicite la relation entre biche et bicheteau, ou encore s.v. Gorge, « De Gorge nous avons Gorgerette \& Gorgias, qui est le collet dont les femmes couurent leurs poictrines. Et si tu veux à l'vn ou à l'autre bailler des epithetes, aide toi de ceux qui sont attribuéz à Collet. », qui présente les substantifs (dans la même taille de caractères que la suite du commentaire) et les définit, mais ne les traite pas localement, renvoyant à une liste d'épithètes fournie dans l'article Collet (cf. § 5.2).

\subsubsection{Adressages subordonnés à patrons rédactionnels réguliers}

Les substantifs présentés dans les 35 composants d'adressage subordonné à patrons rédactionnels réguliers sont des diminutifs de ceux en adresses principales et ils sont associés à des traitements sous forme de listes d'épithètes. L'adressage de ces diminutifs est régulier dans son principe, mais pas complètement dans sa forme textuelle : l'introducteur le plus récurent est « Le dim. » (29 occurrences) et il est le plus souvent suivi de la présentation du diminutif et d'un point final ${ }^{8}$ de composant d'adressage ( "Le dim. Aduocasseau, »s.v. Aduocat, «Le dim. Aignelet. » s.v. Agneau ou Aigneau), mais dans 5 cas l'adressage subordonné concerne plusieurs substantifs. Deux ou trois peuvent être coordonnés par un « \&» (« Les dim. Oiselet \& Oisilon. » s.v. Oiseau ou Oisel, « Les dim. Vignette \& Vignolette. » s.v. Vigne, et « Le dim. Cuueau Cuuette \& Cuuier. » s.v. Cuue ('cuve'), avec un surprenant déterminant au singulier dans le dernier cas, comme s.v. Vipere infra). Deux réalisations graphiques différentes d'un même substantif sont coordonnées par un « ou » ("Le dim. Perdereau ou Perdreau. » s.v. Perdris). Et deux paires de diminutifs liés par un «ou » sont coordonnées par un «\& ( « Les dim. Ourson ou Oursillon, \& Ourset ou Ourselet. » s.v. Ours). L'introducteur « Le dimi. » est employé une fois pour introduire un seul substantif («Le dimi. clouët. » s.v. Clou). L'emploi du troisième introducteur, " dimin. ", peut se faire dans les mêmes patrons de rédaction que précédemment pour présenter un diminutif seul (« Le dimin. Corbeillon.» s.v. Corbeille et «Le dimin. Pucelette. » s.v. Pucelle) ou deux coordonnés par «\&» («Les dimin. Femmette \& Femmelette. » s.v. Femme et « Le dimin. Vipereau \& Viperillon. »s.v. Vipere). Dans un cas de coadressage principal, l'introducteur incluant « dimin. » prend une forme plus textualisée afin de relier le diminutif à l'un des deux substantifs coadressés (« Le dimin. de Chanson est Chansonnette. » s.v. Chant \& chanson).

Le traitement des diminutifs donne lieu à une liste d'épithètes mais pas à des commentaires, même si ceux-ci sont placés après cette dernière, comme c'est le cas s.v. Ours (« L'ours parie au commencement de l'hiuer, tenant l'Ourse embrassee comme font l'homme \& la femme, laquelle aiant chargé se retire à part en sa taniere, ne portant que trente jours,\& faisant le plussouuent cinq petis Oursons : [...] Au surplus ils ont la teste aussi tendre que les lions l'ont dure,\& sont fort suiets à auoir la veuë trouble. »), Perdris (" Pline raconte des choses incroiables de la grande lasciueté de cet oiseau, lequel couure ses œufs d'vne legere terre \& menuë, \& ne le couure jamais ou il les aura premierement faits [...] Quand la Perdris n'a point de petis, \& qu'elle n'est en crainte, elle se couche en vne raie de charrue à la renuerse, \& se couure d'vne motte de terre, qu'elle tient avec ses ongles pour reposer à son aise. On dict qu'elle vit seize ans. », avec la note marginale «Pline liu. 10. chap. 33. » en vis-à-vis de la première phrase), Table (« Ce mot a diuerses significations: mais ici est prins pour ce que les Latins appellent, Mensa. Du Bellai en son Poëte courtisan la nomme,Escole de la Cour. »"), Vent ( « Le Vent n'est autre chose que l'air esmeu, laquelle esmotion aduient en plusieurs sortes. [...] Outre ces quatre Vents principaus y en a quatre autres moiens ou moitoiens, nommés par noz mariniers François Suest, Northest, Northouest, Suouest. ») et Vipere (« Entre les serpens la Vipere fait son fruit parfait \& en vie, aiant premierement fait ses oeufs en son ventre. [...] Au reste ce bestiail aime fort le vin de son naturel, tellement que ceux qui le veulent prendre mettent des pots de terre pleins de vin aupres des haies \& buissons, pource qu'incontinent il se lance dans iceux \& s'eniure. »). Dans chacun de ces cas, le commentaire ne porte pas spécifiquement sur le diminutif, malgré son placement immédiatement après la liste d'épithètes de celui-ci : il exprime des connaissances ou des lieux communs portant sur le référent de l'item en adresse principale, sans pour autant que les référents des diminutifs soient exclus de la portée du commentaire. 
Afin de les en exclure, La Porte opte une fois pour un placement du commentaire avant l'adressage subordonné, s.v. Vigne : « Vigne. Torse ou tortice, feconde, utile, [... I] Aucuns l'appellent Gros navet, les autres feu ardent. [П] Les dim. Vignette \& Vignolette. leune, nouuelle ou renouuelee. ».

Enfin, s.v. Aduocat (cf. fig. 3), une note marginale (cf. § 2) débute à la deuxième ligne de la liste d'épithètes du diminutif aduocasseau et il s'avère difficile de l'interpréter. Elle indique que « Auiourdhui le frãçois ortographe admet raremẽt les diphtõgues, de sorte que celui qui en use indifferãmẽt ne doit estre accusé d'ignorãce. » et est placée en vis-à-vis des articles Aduocat, Faque ou Eaque et Fgine. Porte-t-elle sur la finale d'aduocasseau ou sur la ligature à l'initiale de ceaque ou d'cegine? Son rattachement à un article (voire un sous-article) ou à un autre dépend de la réponse à cette question.

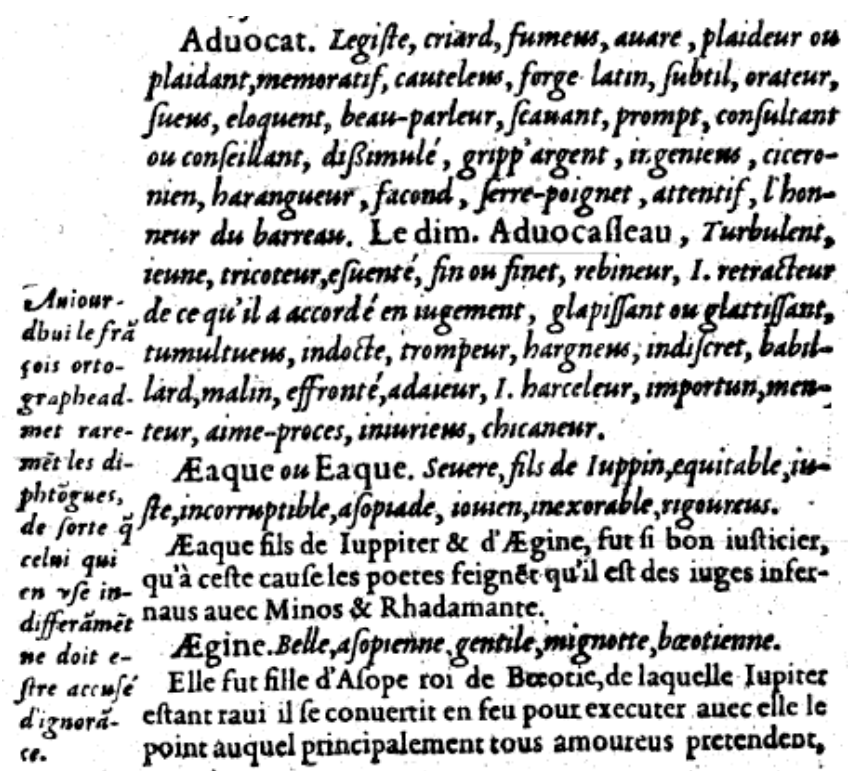

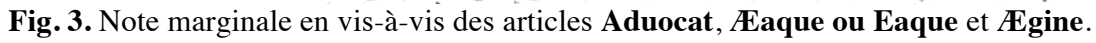

Les traitements inclus dans les articles subordonnés, ceux consacrés aux diminutifs, sont donc moins complexes que ceux des articles principaux, puisqu'ils ne contiennent qu'une liste d'épithètes ou un substitut (s.v. Gorge) et que l'un est peut-être porteur d'une note marginale (s.v. Aduocat). Une question se pose néanmoins : en prenant le soin de distinguer les épithètes des substantifs en adresses principales et celles de ceux en adresses subordonnées, mais en réunissant les deux articles en un, La Porte a-t-il voulu marquer que les épithètes sont mieux partageables entre un substantif et son diminutif qu'entre deux substantifs (aléatoires) traités dans deux articles principaux autonomes?

\subsection{Adressages microstructurels}

Les adressages microstructurels sont des traitements d'unités linguistiques par leur seule mention au sein des articles. Ils n'appartiennent pas à la macrostructure du dictionnaire (les adressages principaux et subordonnés), mais aux traitements des adresses macrostructurelles au sein des articles, ceux-ci débordant de leur programme d'information, puisque ce n'est plus de l'item en adresse qu'il est question, mais d'autres qui ont quelque chose à voir avec lui.

Les Epithetes comptent 162 adressages microstructurels. L'un concerne un féminin (« Au fem. nous disons Patrõnesse. » s.v. Patron), les autres des diminutifs.

Sur les 161 composants d'adressage microstructurel de diminutifs (152 avec « dim. », 2 avec « dimi. » et 7 avec « dimin. », le plus souvent placés à la fin de la liste d'épithètes ${ }^{10}$ ), 42 comportent deux ou trois substantifs coordonnés par «\&» et 7 par « ou ». Les formulations 
sont souvent très voisines de celles des composants d'adressage subordonné (cf. § 3.2). Un cas particulier se détache cependant, celui observable s.v. Cane ou Canart, dont les items en coadresses principales sont les noms de la femelle et du mâle d'un même animal et dont les deux diminutifs répercutent la distinction de genre à relier à celle de sexe, mais en inversant l'ordre de mention: «Les dimin. Caneton \& Canette. ».

Les 4 composants d'adressage microstructurel qui ont un texte différent peuvent soit n'être que des reformulations sans valeur ajoutée ( « II a deux dim. Archelet \& Archerot. » s.v. Archer), soit spécifier à laquelle des coadresses principales se rattache(nt) le(s) diminutif(s) introduit(s) (« Le dim. de fort est fortelet.» s.v. Fort, Fortesse ou Forteresse, « De vieil on tire deux dim. Vieillot \& Vieillote. » s.v. Vieil ou Vieillard et «Hache a pour son dimi. Hachette, », en italique comme les épithètes qui précèdent, s.v. Hache \& Halebarde). Les traitements des sousadresses et des adresses microstructurelles sont donc proches, à la liste d'épithètes spécifiques près toutefois. Aucun des composants d'adressage microstructurel ne fournit d'autres informations que la relation avec ce qui figure en adresse principale (féminin ou diminutif de...), rien qui porterait sur les items introduits ou sur leurs référents.

\subsection{Remarques conclusives concernant les adressages}

La présence, pour les diminutifs, de 161 composants d'adressage microstructurel et de 35 composants d'adressage subordonné introduisant une liste d'épithètes marque bien que, pour La Porte, il était important de fournir cette liste pour certaines unités lexicales, mais que cela est inutile ou de moindre utilité pour la majorité des diminutifs. Ce choix renforce l'idée que la liste d'épithètes introduite par le composant d'adressage principal est héritée par les diminutifs en adresses microstructurelles, et donc vraisemblablement aussi par ceux en adresses subordonnées. Il reste toutefois, dans cette hypothèse, à comprendre pourquoi La Porte a coadressé « Able ou Ablette. » et « Faucille ou Faulx. », en mettant en outre le diminutif en première position dans le second cas (cf. § 3.1.2).

Pour les premières coadresses, un élément de réponse se trouve certainement dans le commentaire de l'article ( « L'Able ou Albe, est vn poisson de riviere de la grandeur du doigt, lequel pour sa gourmandise est facilement prins à l'hameçon. »), les noms able, albe et ablette semblant pouvoir être alternativement employés pour désigner le même petit poisson. Si l'étymologie actuelle de faucille indique que c'est une petite faux (" bas lat. falcicula, dimin. de falx, falcis " faux ». » s.v. faucille dans le Grand Robert de la langue française), l'article des Epithetes ne comporte qu'une liste d'épithètes ( « Croche ou crochue, dentelee, sciante, feneuse, saturnienne, tortue ou tortueuse, mordante, enroüillee, courbe, pointue, crenelee, moissonniere, ravissante, ferree, ague, taillandiere, estivale, recourbee, habile. »), dont chacune semble pouvoir s'appliquer à chacun de ces deux noms d'outils (le premier, le petit, manipulé à une main, et le second, le grand, manipulé à deux mains et avec un ample mouvement du corps).

Il semble donc y avoir coadressage quand le diminutif désigne le même référent que le substantif initial ou un référent qui peut recevoir au même titre les mêmes qualifications, alors qu'il y aura adressage microstructurel quand les diminutifs sont moins sujets à recevoir les qualifications, mais qu'elles sont néanmoins valides pour eux aussi, et sous-adressage quand les diminutifs peuvent recevoir des qualifications qui leur sont plus propres.

\section{Commentaires}

1359 articles des Epithetes comportent un commentaire. Il est souvent terminal et constitué d'un paragraphe (comme s.v. Auerne en fig. 1), mais il existe 1 cas de commentaire non terminal (s.v. Vigne), placé avant le sous-article consacré au diminutif (cf. §3.2.2), 3 cas de commentaires de deux paragraphes (s.v. Amour, cf. $\S 4.2$, Montgibel, cf. $\S 5.2$, et Febue ${ }^{11}$ ) et 15 cas de commentaires entretenant des relations plus complexes avec le reste de l'article. 
Quand les commentaires ne constituent qu'un paragraphe terminal, leur teneur peut être définitoire, comme s.v. Ame ( « Ce mot a diverses significations, quelquesfois se prend pour la vie, aucunefois pour le soufle dont l'homme respire : Signifie aussi le cueur, courage, \& affection : Pareillemẽnt l'esprit de l'homme duquel il vit, \& se prend aussi pour la volonté qui est coniointe avec l'intelligence. »). Mais elle est souvent encyclopédique. Les connaissances formulées concernent des animaux, comme s.v. Ours, Perdris ou Vipere (cf. § 3.2.2) ou encore Able ou Ablette (cf. § 3.4), des plantes (leurs vertus thérapeutiques notamment), des lieux, comme s.v. Auerne (cf. fig. 1), des personnes, comme s.v. Aaron ( « Aaron fut aisné fils de Amram, \& estoit trois ans plus vieil que son frere Moyse, duquel il fut coadiuteur pour supporter l'imperfection de sa lãgue. Apres le trespas d'icelui Aarõ, son fils Eleazare succeda à sa dignité sacerdotale. »). Les informations peuvent aussi être des synthèses de ce que des auteurs ont énoncé à propos du référent du substantif en adresse, comme s.v. Acheron («Les Poëtes disent qu'Acheron est fils de Ceres, \& qu'il n'a point de pere : Mesmes qu'estant enuoié aux enfers il devint fleuue. De luy les ames sont appellees Acherontides \& Acherontees. $»^{12}$ ), ou des renvois vers des textes (cf. § 5.1). Souvent La Porte mêle des avis personnels aux informations, comme s.v. Abricot ( « Ce fruict est plaisant \& delicieus, mais fort suiet à la gelee, \& l'arbre qui le porte dure bien peu. »), ou bien il fait l'éloge ou la critique de personnes connues, comme s.v. Ronsard (« La tresdocte \& non vulgaire poësie de M. P. de Ronsard gentilhomme Vandomois, au commencement des ignorans abbaiee, est aujourd'hui d'vn chacun si fauorablement receuë, signamment de ceux qui sçauent dextrement manier le baston poetique, qu'il n'y a celui qui ne le confesse estre le prime entre tous les poetes François. Et veux aussi que tu sçaches, Lecteur, comme ses oeuures ont esté la principale cause de la peine que i'ai prise à te faire ce recueil d'epithetes, aiant trouvé vn plaisir incroiable à sa naïve façon de les accommoder. »), ou qui lui sont proches, comme son frère s.v. De la Porte (« Ambroise de la Porte Parisien mon trescher aisné frere (duquel ie ne puis sans douleur me representer le trespas) outre la connoissance qu'il auoit des bonnes lettres, il sçauoit tant heureusement rediger par escrit ses mentales cõceptions, que si les trois sœurs inexorables ne lui eussent auant le temps couppé le filet de sa vie, il eut forcé noz suruiuans nepueus de le mettre au nombre des personnes qui ont enrichi la langue Françoise. »). Enfin, les commentaires des noms épicènes comportent l'indication de cette propriété linguistique, soit isolément, soit dans une phrase terminale apposée aux informations précédentes : «Ce mot est masc. \& fem. », s.v. Caresme, Coche, Horloge, etc., ou « Ce mot est masc. \& femin. », s.v. Absince ou Absynthe et Escarboucle, ou encore « Ceste diction est prinse tant au masc. que fem. genre. », s.v. Alarme.

\subsection{Commentaires structurant les épithètes en sous-listes}

Comme nous l'avions observé (Gasiglia \& Pouey-Mounou (2017, § 1.2.)), les commentaires s.v. Amoureuse ou Amante servent à organiser la liste d'épithètes en sous-listes :

« Amoureuse ou Amante. Belle, rusee, [...] infidele, musquee.

Ce sont à mon iugement les mieux conuenables epithetes qu'on puisse donner à ces foles amoureuses, iaçoit que de la plusgrãde partie noz amoureus trãsis n'aient accoustumé d'vser en leurs escrits, principalement vers celles dont ils esperent obtenir quelque faueur, lesquelles ils appellent

Deesses, angelettes, [...] basme, violette:

Et quand ils veullent exprimer les contraires passions d'amour, ils vsent de ces mots, Douce-contraire, douce-ennemie, [...] belle-rebelle, humble-fiere:

Mais si madame l'Amoureuse connoissant possible le maigre proffit qu'on peut tirer de telles personnes, de qui la monnoie est ordinairement en papier, fait la sourde oreille à leurs requestes, elle sera lors nommee,

Ingrate, cruelle, [...] mauvaise, facheuse. »

comme cela est aussi le cas s.v. Aureille ou Oreille :

« Les Epithetes qui s'ensuiuent sont propres aux bestes brutes seulement:

Pendantes, couppees ou decoupees, tendres, fendues, chauvissantes, baissees, longues, creuses, velues. "

s.v. Bruit, qui offre en plus un renvoi vers un article (cf. $\S 5.2)$ :

« Pareillement Bruit signifie Renom \& est prins en bonne \& mauuaise part :

Fameus, commun, populaire, notable, espars, semé, espandu, porte-nouuelle, diuulgué, leger, celebre. VOI RENOMMEE » 
s.v. Choeur (dans la dernière liste, nous observons « \& autres semblables » qui n'est pas une épithète mais une indication de liste incomplète ${ }^{13}$ ) :

« Choeur proprement signifie l'assemblee qui voient iouër les ieux, ou de gens qui dansent: Mais les poetes ordinairement entendent par ce mot la compagnie des Muses, \& lui donnent aussi les epithetes qui leurs [ $\mathrm{sic}]$ sont particuliers: comme,

Sacré, aonien, parnaßide ou parnaßien, chantre, diuin, castalide, musical, celeste, pegasin

s.v. Coucou ou Cocu :

ou pegasien, \& autres semblables. »

«Cet oiseau à la teste semblable à vn pigeon, \& son pennage retire en couleur à celui de l'Esperuier. II change de figure en certain temps de l'an, \& de voix par fois, ponnant tousiours au nid d'autrui, pour la haine que luy portent les autres oiseaus. De là est aduenu que non seulement ceux lesquelz abusent des femmes d'autrui, mais aussi les maris abusez sont appellez Cocus : Tellement que ce nom estant actif \& passif, nous disons Cocu cocuant, \& Cocu cocué. En ceste signification donne lui tels epithetes :

Sot, infame, vil, double-ian, marié, ienin, badaut, desplaisant, hué, siflé, moqué, ian-ian, niais, simple, lascif, mesprisé. »

s.v. Palais :

«Quand ce mot sera pris pour le lieu auquel on plaide, tu t'aideras des epithetes qui s'ensuiuent.

Enroué, tumultueus, bruiãt, criard, miserable, tempestueus, embrouillé, succe-bourse, litigieus, empreßé. »

\subsection{Commentaires contenant des citations}

La Porte peut aussi interpeller le lecteur, comme il le fait s.v. Amour, pour lui demander rhétoriquement s'il veut savoir ce qu'est l'amour, puis il cite Ronsard et termine l'article par un second commentaire qui signale la polysémie d'amour, qui peut être synonyme d'amitié :

«Bref veux-tu sçavoir que c'est qu'Amour?

Amour est un gouffre de maux:

Amour affole le plus sage:

Amour n'est sinon qu'une rage:

Amour aueugle les raisons:

Amour renuerse les maisons:

Amour honnit la renommee:

Amour n'est rien qu'une fumee.

Quand Amour signifie Amitié, il est prins en bonne \& mauvaise part, \& est masc. \& fem. »

L'organisation est comparable s.v. Thebaides, avec un commentaire introducteur de citation et une prolongation de commentaire à teneur bibliographique placée après celle-ci :

«Pris aux ieux rustiques de Du Bellai, où il escrit à Bertrand berger.

Et ces facheuses Thebaides,

Ou n'i a vers sur qui ses doigts

On n'ait rongé plus de cent fois.

Plusieurs ont composé des Thebaides, \& entre autres le poëte Stace Papinien,lequel en

12. liures a diuinement escrit la guerre de Thebes. $\gg$

Nous observons par ailleurs une citation introduite par un commentaire s.v. Guerre :

« Ces epithetes font asses connoistre quels sont les fruits que la guerre produit : toutesfois

i'ai bien encores voulu adiouster les vers qui s'ensuiuent, afin que le lecteur sçachant nos longues ciuiles guerres il desplore auec moi le miserable estat auquel est maintenant toute la France reduitte.

Par la cruelle guerre on renverse les villes,

On depraue les loix divines \& civiles,

On brusle les autels, \& les temples de Dieu,

L'æquité ne fleurit,la justice n'a lieu,

Les maisons de leurs biens demourent despoüillees,

Les vieillards sont occis, les filles violees,

Le pauure laboureur du sien est deuestu,

Et du vice execrable on fait vne vertu. » 
s.v. Ialousie :

« Ce seroit chose trop prolixe à descrire les maux qui procedent de la ialousie: A ceste cause ie t'aleguerai seulemẽt quatre vers de la Peruse, disant ainsi.

Les ennuis continuels

Qui bourrellent nostre vie,

Ne sont pas tous si cruels

s.v. Nil ou Nile :

Que la seule ialousie. »

s.v. Proces :

« Le Nil (fleuue tant renommé) separe l'Affrique d'auec l'Asie, \& vers les parties australes arrouse le païs æthiopiẽ, duquel aussi il sort \& y prend source, puis faisant sa course vers le North, laue le païs ægiptien, le foisonnant par ce gras arrousement d'vne esmerueillable fertilité. Finablement se va lãcer en la mer, s'engoulphãt par sept bouches, ainsi que tesmoigne nostre grand poete Ronsard,disant.

Que par sept huis dedans la mer se vire,

Mesmes sa source aux hommes n'aparoist

Aux plus chauds mois, \& d'une eau limonneuse

Rend à foison l'Egipte bien heureuse. »

« le ne sçai si l'insupportable paresse \& avarice de ceux qui sont i[n]strumens des iugements, ou la frauduleuse malice des parties litigantes, fait que ceste monstrueuse beste de proces regne auiourd'hui de telle sorte en nostre France, mais tant i a que quand on pense voir la fin d'vne premiere action intentee, deux, trois, \& quatre autres renaissent. Qui sera cause qu'à ce propos ie te reciterai ce que lon a facetieusement escrit d'vn païsan, lequel requeroit vn Arrest chastré.

$\checkmark n$ bon vieillard aux grands iours demandoit

Qu'on lui baillast Arrest chastré \& court:

Alors la Court qui ce mot n'entendoit

Le fait venir, \& le bon-[h]omme i court.

Qui leur a dit, l'eu pieça de la Court

$V n$ bel Arrest \& de longueur si grande,

Qu'engendré m'a de proces telle bande,

Qu'impoßible est parfournir au surplus:

Voila, Meßieurs, pourquoi ie vous demande

ou encore s.v. Zoïle :

Arrest chastré qui n'en engendre plus. »

« Zoile fut vn sophiste du temps de Ptolemee, lequel par ses escrits impudens pensoit acquerir grand bruit en reprenant Homere le prince des poëtes:tellement que tous enuieus, \& qui pour blasmer autrui s'en veullent faire accroire,sont maintenant appellez Zoiles. De la dent venimeuse desquels ie prie à Dieu n'estre iamais attaint,\& qu'à toi, Lecteur, soit agreable ma bonne affection, laquelle volontiers me feroit voler plus haut, si les ailes de sçavoir estoient iointes à mon esprit, que tu connoistras neantmoins s'estre en ce labeur laborieus laborieusement emploié : Mais

Comme une femme apres l'enfantement

De son travail n'a plus de souvenance:

Le mal außi que mon entendement

A pour cet œuvre enduré longuement,

L'aiant produit est mis en oubliance. »

Mais, dans d'autres cas, les citations ne sont matérialisées que par une mise en italique au sein du commentaire ${ }^{14}$, comme s.v. Carquois («Pris de Du Bellai, Besoin n'aura du fidelle carquois. ») ou même ne sont pas matérialisées du tout (pas de saut de ligne, pas de mise en italique), comme c'est le cas $s . v$. Fternité ( « Ronsard en l'hymne qu'il a composee de l'Æternité, l'apelle Deesse au grand œil tout-voiant : Roine \& maistresse des ans, des siecles, \& de l'aage. ») et Lentisque (« Du bois de l'arbre Lentisque on fait des Cure-dens, que lon appelle semblablement Lentisques. Or pource qu'ils font ordinairement sortir quelque ordure des dens,nostre Ronsard les appelle escumeuses \& baueuses. ») pour les citations explicitement prises de Ronsard, ou s.v. Surdesse ou Surdité (« Du Bellai la nomme Nourrice de sagesse \& de raison. ») et Table ( « Ce mot a diuerses significations: mais ici est prins pour ce que les Latins appellent,Mensa. Du Bellai en son Poëte courtisan la nomme,Escole de la Cour. ») pour celles de Du Bellay, ou encore s.v. Sibylles pour une double citation de Varron et de Virgile (« Les Sibylles predisoient les choses futures: \& Varron en nõbre dix, assavoir la Persiẽne, Libyenne, Delphiẽne, Cumee, Erythree, Samiẽne, 
Cumaine, propremẽt dite Amalthee, \& par Vergile Deiphobe, Hellespontienne, Phrygienne,\& Tyburtine autrement nommee Albunee. »).

\subsection{Remarques conclusives sur les commentaires}

Environ un tiers des articles comportent un commentaire et souvent ceux-ci sont assez consistants, riches en informations sur les référents nommés ou en lieux communs référés à des auteurs ou les citant. La Porte donne donc une fonction pédagogique à ce composant d'article. Mais, dans 6 articles (s.v. Amoureuse ou Amante, Aureille ou Oreille, Bruit, Choeur, Coucou ou Cocu et Palais), il exploite ce composant pour organiser ses listes d'épithètes. Pour les 5 derniers, ce n'est pas la longueur des listes qui peut motiver cette structuration.

\section{Renvois vers des textes externes ou des articles des Epithetes}

La Porte insère des renvois de diverses natures dans les articles des Epithetes. Quand ils sont externes, ils signalent les auteurs que La Porte souhaitait valoriser auprès de ses lecteurs. Quand ils sont internes, ils mettent en relation des substantifs, souvent des synonymes, afin de mutualiser les épithètes de l'article cible ou, dans de rares cas, à partir d'un commentaire, afin de partager les informations de son commentaire en complément de celles fournies localement.

\subsection{Renvois à des textes externes}

Les renvois peuvent orienter vers des textes de la Pléiade, comme dans le commentaire postposé aux épithètes s.v. Abeille («Abeilles, Aueilles ou Auettes, sont mouches à miel, lesquelles on appelle filles du Ciel, parce que la plus douce partie de leur miel coule du Ciel. Voi Pline liure xj.depuis le v.chap. iusques au xxj. »), Alcine ( "Voi Muret en ses commentaires du premier liure des Amours de Ronsard sur le sonet qui commence,Entre tes bras. »), Basilic ou Basilique (« La diuersité de ce qu'on a escript du Basilic, en fait estimer l'histoire fabuleuse. On dit qu'il est iaunastre, \& a cõme trois petites enleueures sur la teste: Que par son regard \& soufle seulement il tue ceux qui le regardent \& oïent, mesmes fait mourir tous les animaus qui le touchent, encores qu'il soit mort. Bref il n'i a beste plus nuisible sur la terre,iaçoit qu'il n'ait pas vn demi pied de longueur. Pour en sçavoir davantage voi ce que dit Pline liure 8. chap.21. »), Cytise (« Plusieurs ont estimé que le Cytise fut vne herbe, mais ceux qui en ont eu vraïe cognoissance, disent qu'il est arbrisseau grand comme le Meurte. Voi Matthiole au chap. 108.de ses commentaires sur le 4. liure de Dioscoride. »), Nenufar ou Neufar ( «Ceste herbe croist és marez, \& és eaux qui sont à requoi, aiant les fueilles fort larges, \& toutes rõdes, dont les vnes nagent sur l'eau, \& les autres se nourrissent au fonds de l'eau : de laquelle herbe il y en a qui a la fleur jaune, aiant d'autres proprietés, que n'a celle qui l'a blanche. Voi Matthiole en ses commentaires sur les 131. \& 132.chap.de Diosc. liu. 3. ») et Prelat (« Si tu veux auoir connoissance de plusieurs autres qualitez requises au Prelat, Voi sainct Paul au commencement du 3. chap. de sa I. Epistre à Timothee. »).

\subsection{Renvois vers des articles des Epithetes}

La Porte propose régulièrement des renvois vers d'autres articles afin de fournir des épithètes complémentaires déjà inventoriées pour d'autres substantifs. Ils sont alors placés en fin de liste d'épithètes, comme s.v. Bastilde, Bastille ou Bastillon (« Forte, imprenable, asseuree. VOI CHASTEAV ET FORTERESSE. »). Si, dans le cas d'un renvoi à un texte extérieur, l'introducteur « Voi » n'a de majuscule qu'initiale, dans les renvois internes au dictionnaire, « VOI » et l'adresse de l'article cible sont en majuscules dans 172 occurrences, contre 17 où ils n'ont que des majuscules initiales. Ces renvois vers des articles ne sont pas accompagnés d'une remarque, sauf s.v. Adolescence («Indiscrete, sotte, [...] superbe, voluptueuse. VOI IEVNESSE, à qui lon donne communément les Epithetes propres à l'Adolescence. ») et Berecynthe («Mere des dieux, phrygienne. VOI CIBELE, laquelle a prins ce nom d'une montaigne de Phrygie nommee Berecynthe. »), ou, avec un saut de 
ligne séparant la liste d'épithètes du renvoi, s.v. Cheueleure (« Crineuse, gente ou gentille, lascive. [T] Voi les epithetes des Cheveus, ausquels noz poëtes attribuent ce qui est autant ou plus propre à Cheuelure. »).

Quand il y a un renvoi et un adressage microstructurel (pour un diminutif - 3 occurrences), ce dernier est placé soit après le renvoi, s.v. Tresse (« Ondoiante, secoüee, [...] mignarde, luisante. VOI CHEVEVS. Le dim. Tressette. ») et Truye ou Truie (« Grosse ou grasse, feconde, [...] famelique ou fameilleuse, marche-tard. VOI PORC. Le dim. Truiette. »), soit avant, s.v. Coque ou Coquille (« Ouale, tendre, creuse, torte, aspre, grumeleuse, fraile, ronde, bossue, raboteuse, tortisse, polie. Le dim. Coquillon. VOI CONQUE. »).

Dans 5 cas les renvois ne semblent pas être faits vers les listes d'épithètes mais vers les commentaires des articles, puisque La Porte emploie « Voi ce que i'ai dit/escrit» et que ces propos sont tenus dans un paragraphe autonome placé après la liste d'épithètes, dans la position des commentaires : s.v. Cinnamone ou Ciname («Voi ce que i'ai dit de Canelle. »), Deiphobé («Voi ce que i'ai dit de Cumee. »), Montgibel (en $2^{\mathrm{e}}$ paragraphe : " C'est vne montaigne de Sicile nommee par les vieux Latins Ætna, laquelle vomit perpetuellement de grosses flammes de feu. [T] Voi ce que i'en ai dit parlant d'Ætne. »), Philomele ou Philomene (« Voi ce que i'ai escrit de Teree, pour entendre comment Philomele fille de Pandion roi d'Athenes, fut changé [sic] en Rossignol. ») et Progné (« Pour euiter vne redite, voi ce que i'ai dit de Teree, où tu apprendras pourquoi Progné fut changé [sic] en hirondelle. »).

Il serait toutefois réducteur de ne prendre en compte que les renvois faits au moyen de l'opérateur « Voi » / « VOI », La Porte employant aussi « Aide toi » dans 3 commentaires pour renvoyer aux épithètes des articles cibles : s.v. Chantre (« Aide toi des epithetes baillez a Chant \& Chanson. »), Gorge (« De Gorge nous avons Gorgerette \& Gorgias, qui est le collet dont les femmes couurent leurs poictrines. Et si tu veux à I'vn ou à l'autre bailler des epithetes, aide toi de ceux qui sont attribuéz à Collet. », cf. §3.2.1) et Veuë (« Aide toi des epithetes attribuees aux ïeux.»), et «CHERCHE » s.v. Auette (« CHERCHE ABEILLE. », cf. fig. 1).

\section{Conclusion}

Au terme de cette analyse structurelle, nous avons progressé dans la connaissance du dictionnaire de combinaisons de mots de La Porte et, ce faisant, nous avons amélioré notre capacité à le baliser finement.

Nous savions que le patron régulier des articles consiste en la présentation d'un substantif (nom commun ou nom propre) en adresse suivi d'une liste d'épithètes puis d'un commentaire optionnel. Nous avons observé en outre que les noms communs peuvent être désambiguïsés (en particulier quand il y a des homographes à la nomenclature), ce qui fait qu'un segment de texte codé typographiquement comme une adresse principale d'article peut être à décomposer en un substantif (un élément $\langle w>$ porteur d'un attribut @type de valeur "subst") et un sélecteur de sens (que nous balisons avec un élément <glose>). Dans les 4 cas de coadressages avec déterminatif (Bourse ou Boursette au pasteur, Materas ou Mattelas a coucher, Palme ou Paulme de la main, Tuyau ou Tuiau de bled), il convient de procéder de même, mais en distribuant le sélecteur final sur chaque substantif. Pour les recherches dans les textes de la Pléiade des attestations (qui ont vocation à enrichir l'épithétaire) des substantifs associés à des déterminatifs cooccurrents de chacune des épithètes listées à leur suite, il ne faut prendre en compte que les substantifs, pas les sélecteurs de sens (pour Aspic Serpent, Port signifiant contenance, Carriere pour Course, etc.). Néanmoins certaines dénominations polylexicales sont lexicalisées (Instrumens de musique, Mouches à miel, etc.) et doivent être recherchées globalement. D'autres sont lexicalisées dans leur forme polylexicale, mais sont également employées en se limitant au premier nom dans des contextes favorables (Presse d'Imprimerie, Cep de Vigne, etc.). Les recherches en corpus de ces substantifs associés à des déterminatifs dans le composant d'adressage conduisent donc à récolter des attestations de l'homographe quand seul le nom tête est cherché et à ne pas repérer les emplois au sein desquels la 
préposition introduisant le second nom est différente de celle employée par La Porte (par exemple s'il y a carrière à course dans un texte plutôt que pour course).

Quand il y a coadressage, il est nécessaire de bien repérer chaque substantif et de rechercher toutes les attestations de chacun, mais cela ne pose pas de problème particulier. La question est différente pour les sous-adressages. Puisque La Porte a créé des sous-articles plutôt que des articles autonomes, il nous semble qu'il faut prioritairement chercher les attestations des substantifs cooccurrents des épithètes qui leurs sont attribuées, mais que nous devons aussi étendre la recherche aux cooccurrences des substantifs en sous-adresses associés aux épithètes de l'article principal et inversement, et matérialiser, dans l'affichage des données extraites, les extensions de recherches au-delà de ce que La Porte avait signifié en structurant son article. Cette extension des recherches d'attestations vaut donc logiquement aussi pour les adressages microstructurels.

L'analyse des commentaires a conduit à observer la pluralité de leurs portées et de leurs fonctions. Le balisage doit en tenir compte, pour distinguer les commentaires structurants des autres, mais aussi pour permettre aux lecteurs de notre version électronique de faire des recherches dans les commentaires encyclopédiques ou dans ceux où La Porte se réfère à des auteurs ou les cite, par exemple.

Les renvois à des articles doivent, selon leur portée, soit inciter à étendre les recherches de cooccurrences aux épithètes de l'article cible (comme nous devons le faire pour les sousadressages et les adressages microstructurels), soit conduire à afficher le commentaire de cet article en plus de celui de l'article courant.

Les renvois à des textes externes, comme les citations ou les références trouvées dans les commentaires ou dans les listes d'épithètes, invitent, eux, à créer un réseau hypertextuel, qui permettra d'accéder aux textes évoqués (ou au moins à des extraits plus longs) à partir de l'interface du dictionnaire.

La compréhension de l'organisation structurelle des articles des Epithetes conduit donc à mieux appréhender la teneur des connaissances que La Porte a souhaité rendre accessibles à ses lecteurs et à mieux valoriser son texte en l'enrichissant d'attestations de ce qu'il a relevé dans les articles, mais aussi des extensions qu'il a proposées par le jeu des divers adressages subordonnés et microstructurels, des renvois, et des références et citations.

\section{Références}

Bierbach, M. (1989). Les Épithetes de Maurice de La Porte de 1571 : ouvrage lexicographique, encyclopédique et rhétorique. Kremer D. (dir.), Actes $d u$ XVIII ${ }^{e}$ Congrès international de linguistique et de philologie romanes. Tübingen : Niemeyer. T. IV-6, 44-60.

Clark, C. E. (1970). Un dictionnaire des idées reçues du XVI ${ }^{\mathrm{e}}$ siècle : les Épithetes de Maurice de La Porte. Revue des sciences humaines, 138, 187-196.

Gasiglia, N. \& Pouey-Mounou, A.-P. (2017). Lier les cooccurrences des Epithetes (1571) de Maurice de La Porte et celles des textes de la Pléiade. Corela [En ligne], HS-21, 2017, URL : http://corela. revues.org/4830, mis en ligne le 16 février 2017 ; DOI : 10.4000/corela.4830.

Grand Robert de la langue française. CD-ROM version 2.0, Paris : Le Robert - SEJER / Bureau van Dijk. 2005.

La Porte, M. (1571). Les Epithetes. Fac-similé. Genève : Slatkine Reprints. 1973.

Pouey-Mounou, A.-P. (2003). Petite poésie portative : les exercices de style des Epithetes de La Porte. Bibliothèque d'Humanisme et Renaissance, 65, 51-67.

Pouey-Mounou, A.-P. (2005). Les dictionnaires d'épithètes, laboratoires de l'aptum. Jones-Davies M.-Th. (dir.), Culture : collections, compilations. Paris : Champion. 143-160. 
Pouey-Mounou, A.-P. (2008). Grandeur et décadence d'un tout petit genre : les épithétaires de la Renaissance. Dupèbe J., Giacone F., Naya E. \& Pouey-Mounou A.-P. (dir.), Esculape et Dionysos, Mélanges en l'honneur de Jean Céard. Genève : Droz. 1065-1079.

Pouey-Mounou, A.-P. \& Gasiglia, N. (2015). Le lexicographe au travail : le traitement des Hymnes cosmologiques de Ronsard (1555-1556) dans les Épithetes de La Porte. Hache S. \& Pouey-Mounou A.-P. (dir.), L'épithète, la rime et la raison. La lexicographie poétique en Europe aux XVI ${ }^{e}-X V I I^{e}$ siècles. Paris : Classiques Garnier. 237-277.

Pouey-Mounou, A.-P. \& Gasiglia, N. (à paraître). La Porte, la Pléiade et Bacchus. Pouey-Mounou A.-P. \& Huchon M. (dir.), Actes des journées d'étude Inqualifiables fureurs : Qualifications des figures de l'inspiration dans l'Europe de la Renaissance, Université Lille 3, 29-30 janvier 2015.

Rouget, F. (éd.) (2009). Les Epithetes, 1571. Paris : Champion.

Text Encoding Initiative. URL : http://www.tei-c.org/Guidelines/P5/.

${ }^{1}$ Cet article a bénéficié des lectures préalables de deux experts anonymes et de Pierre Corbin. Merci à chacun pour leurs suggestions. Il doit également beaucoup au travail à quatre main que nous avons engagé, Anne-Pascale Pouey-Mounou et moi.

${ }^{2}$ Le texte de l'édition électronique est basé sur Les Epithetes, fac-similé publié par Slatkine Reprints en 1973. Les images et citations de cette contribution viennent de la version des Epithetes de Gallica, une autre édition de 1571 .

Le texte original de l'épithétaire est en police à empattements avec de l'italique et deux tailles de caractères, mais, pour une meilleure lisibilité des citations dans cette contribution (dont le texte est en Times New Roman), elles sont en Arial pour les composants ayant une plus grande taille de caractères et en Arial Narrow pour les composants en taille "normale".

Il existe aussi une édition imprimée sans annotation systématique des sources (éd. F. Rouget, Paris, Champion, 2009) et une édition partielle en ligne avec des attestations des paires substantif épithète des articles mais sans sélection de celles-ci (http://www.preambule.net/).

Les Epithetes ont connu quelques études, notamment Clark (1970), Bierbach (1989) et Pouey-Mounou (2003, 2005 et 2008).

${ }^{3}$ Les chiffres fournis dans cette contribution sont ceux des décomptes faits à partir de l'état actuel du XML, mais le dictionnaire balisé est en cours de parachèvement. Une relecture minutieuse du balisage conduira peut-être à en réviser certains de quelques unités.

${ }^{4}$ Pour plus d'informations sur ce dictionnaire et sa place de dans la production du XVI ${ }^{\mathrm{e}}$ siècle, cf. Gasiglia \& Pouey-Mounou (2017, § 1.).

${ }^{5}$ La Porte, Les Epithetes, « Advertissement av lectevr », $3^{\mathrm{e}} \mathrm{p}$.

${ }^{6}$ Le XML (eXtensible Markup Language) est un langage de balisage dit extensible parce que son vocabulaire n'est pas prédéfini (à la différence du HTML), et qu'il peut être défini de manière libre en fonction des besoins des utilisateurs. Des standards, recommandations ou normes régissent cependant les emplois partagés de ce langage de manière à ce que des échanges de documents émanant de différentes sources puissent être envisagés, notamment la Text Encoding Initiative (TEI) pour le secteur de l'édition. Le balisage des Epithetes se conforme aux recommandations de la TEI:P5 mais pas à celles conçues spécifiquement pour les dictionnaires (cf. http://www.tei-c.org/release/doc/tei-p5-doc/fr/html/DI.html), l'emploi d'éléments plus communs devant, nous l'espérons, faciliter les échanges avec d'éventuels partenaires éditoriaux non spécialistes de dictionnaires.

${ }^{7} 2$ substantifs en adresse sont omis et le motif est expliqué en début d'article : Con («Par honnesteté i'ai teu le nom vulgaire de la partie honteuse de la femme, dont les epithetes s'ensuiuent. ») et Vit (« L'honneur m'a commandé ne mettre ici le nom commun du membre honteus de l'homme, mais trop bien ses epithetes. »).

${ }^{8}$ Ce point est exceptionnellement remplacé par une virgule s.v. Aduocat.

${ }^{9}$ La dénomination Escole de la Cour, reprise à Du Bellai, constitue un synonyme de l'item en adresse table. Elle peut être employée dans certains textes de la Pléiade avec les épithètes listées pour table par La Porte. Il est donc intéressant de présenter également les contextes de ses attestations. 
${ }^{10}$ Parfois ce traitement microstruturel est articulé à un renvoi qui figure lui aussi à la fin de la liste d'épithètes (cf. § 5.2), le renvoi étant soit avant l'adressage microstructurel, s.v. Tresse et Truye ou Truie, soit après s.v. Coque ou Coquille, mais il se trouve aussi une fois sur une ligne autonome (s.v. Barbeau : «Les dim. Barbarin \& Barbillon. »).

${ }^{11}$ S.v. Febue : « I. aiant vne petite chose noire au cul, que les Latins appellent hilum. [T] Les febues engendrent ventositez, \& sont autant difficiles à digerer que chose qui soit, estans fresches \& vertes si on les mange elles causent de grandes humiditez au corps. Leur farine appliquee auec vinaigre miellé, a mode de cataplasme, est propre aux apostumes \& inflammations, suruenues par quelque coup : elle fait aussi perdre le laict, \& engarde que le poil ne vient de long temps sur le penil, l'appliquant de ceste façon au bas du ventre des ieunes enfans:davantage elle mundifie les taches de la peau, \& autres taches rousses, comme celles qui sont causees du soleil. ».

${ }^{12}$ On constate aussi, s.v. Acheron, que des dérivés morphologiques peuvent être introduits.

${ }^{13}$ Des indications de listes incomplètes se retrouvent dans une énumération du commentaire proposé s.v. Autour comme dans une de celui qui l'est s.v. Laisard ou Lezard, et dans une remarque introductive du segment alphabétique des mots en $\mathrm{Y}$ (« Les dictions que plusieurs escriuent par y, comme yeuse, yeux, yuoire, yuraïe ou yuroïe, yurõgne, \& autres semblables, tu les chercheras a i, \& yuer à la lettre de $\mathrm{H}$. »).

${ }^{14}$ Deux citations en italique figurent hors des commentaires, en fin de listes d'épithètes :

- s.v. Renommee, l'indication "Vergile l'appelle " après la dernière épithète nous conduit à observer que les listes d'épithètes sont susceptibles d'avoir à leur suite de la métalangue sans matérialisation typographique mais avec des indices graphématiques (le point qui sépare la dernière épithète du renvoi et le fait que celui-ci est suivi d'une virgule alors qu'une liste d'épithètes se termine par un point) :

«Renommee. Publique, messagere, vagabonde, babillarde, enuieuse, cornante deesse, blanche, commune, prompte, legere, tout-uoiante, belle, viste, heureuse ou malheureuse, emplumee, poste du monde, fille du nepueu d'Atlas. Vergile l'appelle,

Monstre superbe, horrible, \& tout plein d'ieux

Qui court leger, \& vole encore mieux.»

- s.v. Quignon, une citation est mentionnée dans une liste d'épithètes (réduite à 1 qualification), pour illustrer l'emploi de celle-ci, et là encore le nom de l'auteur n'est pas matérialisé typographiquement, mais, comme la liste d'épithètes est un singleton, il suit le point final de celle-ci :

«Quignon. Gros. Ronsard, Vn gros quignon buret de pain.» 